\title{
RECONCILIAÇÃO DE DADOS EM COLUNAS DE DESTILAÇÃO UTILIZANDO O SIMULADOR EMSO
}

\author{
MENEZES, D. Q. F. ${ }^{1}$, SARRUF, I. ${ }^{1}$, PEIXOTO, F. C. ${ }^{1}$ e PRATA, D. M. ${ }^{1}$ \\ ${ }^{1}$ Universidade Federal Fluminense, Departamento de Engenharia Química e de Petróleo. \\ E-mail para contato: pratadiego@gmail.com
}

\begin{abstract}
RESUMO - Este trabalho apresenta um estudo sobre reconciliação de dados em estado estacionário em colunas de destilação, com base em dois trabalhos publicados na literatura científica. É realizada uma breve revisão bibliográfica com foco nos problemas que envolvem colunas de destilação. O primeiro exemplo é uma coluna debutanizadora. O segundo exemplo é constituído por sete colunas de destilação de óleo cru, incluindo variáveis não medidas e detecção de erros grosseiros. Os problemas selecionados para estudo são resolvidos no pacote computacional EMSO (Ambiente para Modelagem, Simulação e Otimização) que dispõe de rotinas computacionais propriamente desenvolvidas para reconciliação de dados em estado estacionário com restrições lineares ou não lineares. Resultados satisfatórios são obtidos e o desempenho do pacote computacional EMSO pode ser avaliado e comprovado. Assim, são apresentadas as vantagens da reconciliação de dados e sua contribuição para processos de engenharia química.
\end{abstract}

\section{INTRODUÇÃO}

Medidas de processo precisas são de extrema importância para controle, otimização, qualidade, segurança e eficiência do processo. Entretanto, essas medidas contêm erros, aleatórios e grosseiros, causados, por exemplo, pela imprecisão intrínseca dos instrumentos de medição. Desta forma, não se espera que os dados medidos obedeçam às leis de conservação. Portanto, um procedimento de retificação de dados (RTD) é essencial para recuperar satisfatoriamente a informação contida nos dados. Este é basicamente dividido em três etapas: classificação de variáveis; detecção de erros grosseiros (DEG) e reconciliação de dados (RD).

O procedimento mais utilizado na RTD é a RD, onde dados medidos são ajustados de maneira estatisticamente coerente pelo estimador (função objetivo) resultante da formulação de máxima verossimilhança sobre a distribuição estatística dos erros de medição assumida, de forma a satisfazer às leis de conservação e demais restrições impostas ao sistema (modelo matemático), obtendo estimativas confiáveis para as variáveis e parâmetros do processo (Prata et al., 2010). Tradicionalmente é assumida distribuição Normal, que resulta no estimador de mínimos quadrados ponderados (MQP).

A primeira etapa na RTD é a classificação das variáveis. Esta etapa determina se a informação disponível é suficiente para resolver o problema de reconciliação e identificar os conjuntos de variáveis observáveis (variáveis medidas e não-medidas que podem ser estimadas por meio das demais variáveis medidas e pelas restrições do processo) e nãoobserváveis (variáveis não medidas que não podem ser estimadas). A segunda etapa na RTD é 
a DEG, um tipo especial de erro, que não segue a distribuição estatística de erros assumida. Esses erros podem ser causados por má calibração dos instrumentos de medições, deterioração dos sensores, picos de energia, entre outros. Entretanto, para obter uma estimativa precisa, para parâmetros e variáveis, o efeito da influência negativa de erros grosseiros deve ser minimizado ou eliminado. A literatura tem apontado a utilização de estimadores robustos para reconciliação robusta de dados (RRD) (Prata et al., 2010).

Um dos processos de interesse da indústria química e petroquímica é a destilação. Esta é uma antiga operação unitária, tendo como principais aplicações o fracionamento do petróleo, a obtenção de álcoois, a extração de essências e purificação de substâncias (Kister, 1992). Atualmente, com a evolução dos computadores, modelos matemáticos e projetos de colunas de destilação através de simuladores, como: Aspen/Hysys, ProII, ChemCAD e EMSO, entre outros, tem se tornado uma prática bastante eficaz e precisa, porém, bastante complexa. Isto se deve, em parte, a existência de correlações entre as variáveis de processo, e a utilização conjunta dos procedimentos de RD/DEG, controle e otimização, para fins de aplicação real nestes equipamentos em processos industriais.

Seguindo esta direção, este trabalho apresenta um estudo sobre RD em estado estacionário em colunas de destilação, utilizando o simulador EMSO (Soares e Secchi, 2003).

\section{REVISÃO DA LITERATURA}

A aplicação de RD e DEG em problemas de engenharia química inicia-se nos anos 60, a partir daí muitos artigos e livros aplicados a processos químicos vêm sendo escritos. Entretanto, há um número pequeno de trabalhos de RD em aplicação reais (Prata et al., 2010).

A complexidade de tratar com robustez a RD em um processo não trivial como a destilação juntamente com modelos fenomenológicos rigorosos de colunas de destilação, como por exemplo, as equações MESH - Mass, Equilibrium, Summation and Enthalpy (Kister, 1992), faz com que se tenha uma escassez de artigos neste campo. Geralmente, os modelos são considerados em estado estacionário e representados apenas por balanço de massa, equações de normalização e balanço de massa por componente. Os dois primeiros resultam em sistemas lineares, e o último resulta em um problema não linear mais simples, chamado de bilinear, uma vez que a não linearidade é representada especificamente pelo produto de duas variáveis de decisão para o problema de RD (vazão e concentração).

Rao e Narasimhan (1996) apresentaram um estudo de uma coluna debutanizadora com 8 componentes. Wang et al. (2004) avaliaram uma estratégia baseada na utilização conjunta dos testes de medida e nodal (MT-NT) para RD e DEG em um conjunto de 7 colunas em série. Diferentemente, dos exemplos anteriores, que consideraram problemas em estado estacionário, Farzi et al. (2008) desenvolveram um modelo dinâmico e mais complexo para realizar a RD dinâmica, em uma coluna de destilação, estimando as temperaturas por meio de duas estratégias: redes neuronais artificiais e filtro de Kalman estendido.

O simulador EMSO - Ambiente para Modelagem, Simulação e Otimização - (Soares e Secchi, 2003) dispõe de rotinas computacionais propriamente desenvolvidas para RD em estado estacionário com restrições lineares ou não lineares, incluindo testes tradicionais para a DEG e estimadores robustos como a Normal Contaminada (Özyurt e Pike, 2004). 


\section{O PROBLEMA DE RECONCILIAÇÃO DE DADOS ESTACIONÁRIA}

Comumente, os modelos de processos são representados por balanços de massa e energia e incluem também correlações termodinâmicas, propriedades físicas, relações de equilíbrio e reações químicas. Nesse caso, o modelo matemático do processo resulta em um sistema de equações algébricas não lineares. O procedimento de RD não linear é então baseado na solução de um problema de otimização não linear. O problema de $\mathrm{RD}$, em estado estacionário, é descrito como:

$$
\min _{\underline{x}} \frac{1}{2}[\underline{x}-\underline{z}]^{T} \underline{\underline{V}}^{-1}[\underline{x}-\underline{z}]
$$

sujeito a:

$$
\begin{aligned}
& \underline{h}(\underline{x}, \underline{u})=\underline{0} \\
& \underline{g}(\underline{x}, \underline{u}) \geq \underline{0}
\end{aligned}
$$

onde:

$$
\begin{array}{ll}
\underline{x} & =\text { Vetor das variáveis medidas reconciliadas. } \\
\underline{z} & =\text { Vetor das variáveis medidas. } \\
\underline{\underline{V}} & =\text { Matriz de variância-covariância dos erros das variáveis medidas. } \\
\underline{u} & =\text { Vetor das variáveis não medidas estimadas (observáveis). } \\
\underline{h}(\underline{x}, \underline{u}) & =\text { Vetor das equações de restrições algébricas lineares e/ou não lineares. } \\
\underline{g}(\underline{x}, \underline{u}) & =\text { Vetor das equações de restrições de desigualdade, incluindo limites. }
\end{array}
$$

\subsection{Reconciliação Robusta de Dados (RRD)}

Existem muitas classes de estimadores robustos, sendo as mais populares aquelas utilizadas nos estimadores-M, que são generalizações de um estimador de máxima verossimilhança (Prata et al., 2010). Assumindo que os erros de medição não são correlacionados, o problema de RRD estacionário, de forma generalizada, adota a formulação,

$$
\min \sum_{i} \rho\left(\frac{x_{i}-z_{i}}{\sigma_{i}}\right)=\min \sum_{i} \rho\left(\xi_{i}\right)
$$

sujeito a:

$$
\begin{aligned}
& \underline{h}(\underline{x}, \underline{u})=\underline{0} \\
& \underline{g}(\underline{x}, \underline{u}) \geq \underline{0}
\end{aligned}
$$

onde $\rho$ é uma função razoavelmente monotônica, $\xi_{i}$ e $\sigma_{i}$ são, respectivamente, o resíduo padronizado e o desvio padrão da variável discreta medida $z_{\mathrm{i}}, \underline{x}$ e $\underline{u}$ são os vetores das variáveis medidas reconciliadas e não medidas (observáveis) estimadas, respectivamente. Finalmente, $\underline{h}$ e $g$ são as restrições algébricas de igualdade e desigualdade, respectivamente. 
Apresentam-se os estimadores de MQP e Normal Contaminada (NC) como possíveis escolhas para $\rho$ (Özyurt e Pike, 2004).

$$
\text { MQP: } \quad \rho_{M Q P}\left(\xi_{i}\right)=\frac{\xi_{i}^{2}}{2}
$$

Normal Contaminada: $\quad \rho_{N C}\left(\xi_{i}, b, p\right)=-\ln [(1-p)] \exp \left(-\frac{\xi_{i}^{2}}{2}\right)+\frac{p}{b} \exp \left(-\frac{\xi_{i}^{2}}{2 b^{2}}\right)$

onde:

$p \quad=$ probabilidade de valores espúrios $(\operatorname{com} p<0,5)$.

$b^{2} \sigma_{i}^{2}=$ variância da contaminação por estes erros $(\operatorname{com} b>1)$.

Na Equação 8, b e p são parâmetros de sintonia relacionado à eficiência relativa, sendo calculada em relação a uma distribuição, quase sempre adotada a distribuição Normal. Para uma eficiência relativa de $95 \%$ os valores de p e b são 0.235 e 10, respetivamente.

\section{RECONCILIAÇÃO DE DADOS EM COLUNAS DE DESTILAÇÃO}

Nesta seção são apresentados os estudos de casos em colunas de destilação.

\subsection{Estudo de Caso 1}

O primeiro caso avalia uma coluna debutanizadora com uma corrente de entrada, duas de saída (topo e fundo) e com 8 componentes, proposto originalmente por Rao e Narasimhan (1996) e ilustrado na Figura 1. Os autores consideraram estado estacionário, o balanço de massa por componente e as equações de normalização para as concentrações nas correntes. Isto resulta em um sistema bilinear. Neste sistema não há presença de erros grosseiros, particularmente, existem variáveis não medidas e variáveis consideradas fixas (livre de reconciliação). Os autores comparam a técnica de programação não linear (PNL) com outros dois métodos de solução para sistemas bilineares: a matriz de projeção (Crowe et al., 1986) e o método de Simpson (Simpson et al., 1991), que basicamente visam a eliminação do conjunto de variáveis não medidas da $\mathrm{RD}$, estimando seus valores após a $\mathrm{RD}$ das variáveis medidas. Maiores detalhes são apresentados em Narasimhan e Jordache (2000).

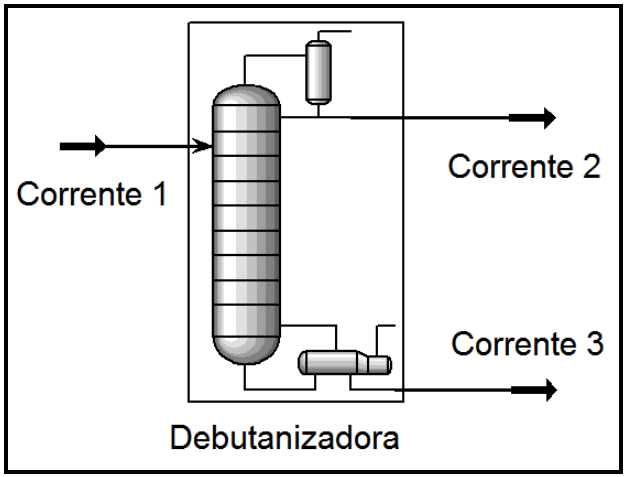

Figura 1 - Coluna debutanizadora (Rao e Narasimhan, 1996). 


\subsection{Estudo de Caso 2}

O segundo caso avalia um conjunto de 7 colunas em série, proposto originalmente por Wang et al. (2004) e ilustrado na Figura 2. Os autores consideraram estado estacionário e apenas o balanço de massa global. Isto resulta em um sistema linear. Neste sistema há presença de múltiplos erros grosseiros, particularmente. Os autores avaliaram uma estratégia baseada na utilização conjunta dos testes de medida e nodal (MT-NT) para RD e DEG.

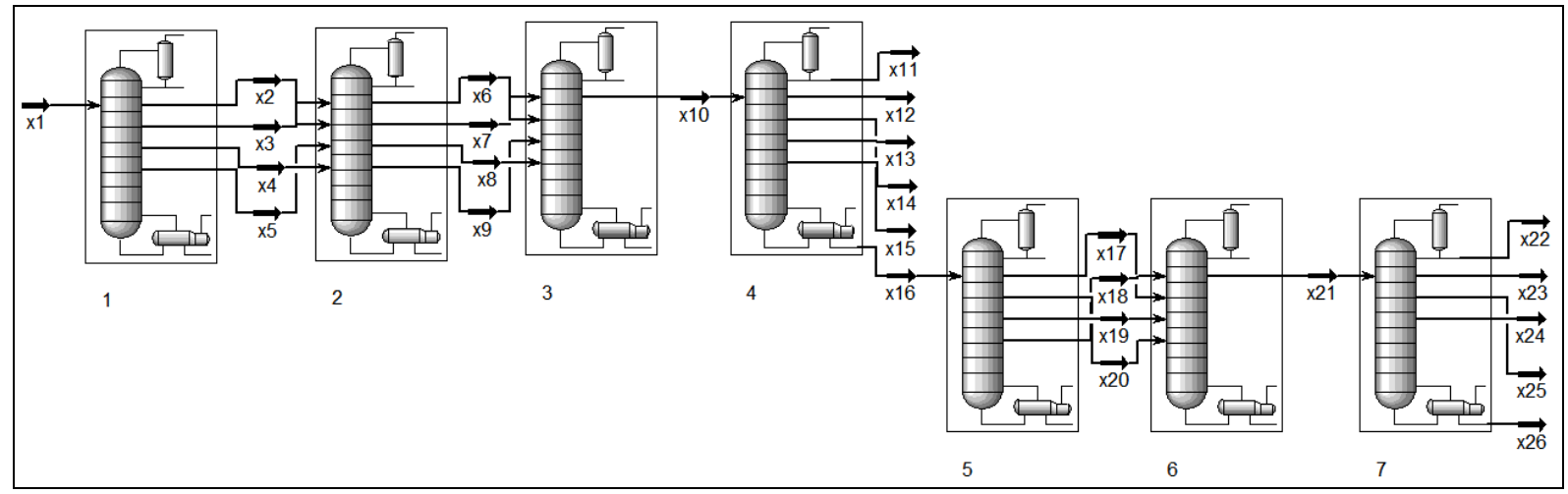

Figura 2 - Fluxograma de destilação de óleo cru (Wang et al., 2004).

\subsection{Critério de Avaliação}

Seguindo a formulação do problema de RD, deve-se minimizar a função objetivo (Fobj) e satisfazer as restrições, que computacionalmente corresponde a minimizar seus respectivos resíduos. Desta forma, são usualmente utilizados os seguintes critérios de avaliação:

Fobj (Função Objetivo): Quando a mesma função objetivo for utilizada no procedimento em $\mathrm{RD}$, independentemente das estratégias utilizadas, quanto menor o seu valor, provavelmente melhor terá sido o resultado, desde que as restrições estejam satisfeitas.

Soma absoluta dos resíduos (SARES): Consiste na soma absoluta de cada resíduo das restrições. Quanto menor o seu valor, provavelmente melhor terá sido o resultado.

SSE (Sum of Squared Error): Consiste na utilização da Equação 9.

$$
S S E=\sum\left(x_{\text {reconcilialo }}-x_{\text {exato }}\right)^{2}+\sum\left(u_{\text {estimado }}-u_{\text {exato }}\right)^{2}
$$

Quanto menor for a SSE obtida, provavelmente melhor terá sido o resultado, indicando uma diferença pequena entre os valores reconciliados /estimados e os exatos. Uma crítica é que este critério não leva em consideração o desvio-padrão para as variáveis medidas (resíduo padronizado).

Os critérios de SARES e SSE podem ser utilizados independentemente da Fobj utilizada (estimador). Desta forma, estes critérios podem ser utilizados para comparação de estimadores robustos e outras estratégias nos procedimentos de RD e DEG. 


\section{RESULTADO E DISCUSSÃO}

\subsection{Resultado do Estudo de Caso 1}

Na Tabela 1 são apresentados os resultados obtidos neste trabalho e por Rao e Narasimhan (1996), bem como os valores medidos e respectivos desvios-padrão.

Tabela 1 - Resultados da RD para o estudo de caso 1.

\begin{tabular}{|c|c|c|c|c|c|c|c|}
\hline & & \multirow[b]{3}{*}{ Medido } & \multirow[b]{3}{*}{$\sigma_{\mathrm{i}}$} & \multicolumn{4}{|c|}{ Valores Reconciliados } \\
\hline & & & & \multicolumn{3}{|c|}{ (Rao e Narasimhan, 1996) } & \multirow[b]{2}{*}{ EMSO } \\
\hline & & & & Crowe & Simpson & PNL & \\
\hline Corrente 1 & Entrada & 9.2200 & 0.0922 & 8.4400 & 8.4100 & 8.4100 & 8.416700 \\
\hline \multirow[t]{8}{*}{ Composição \% } & 1 & 0.0200 & 0.0002 & 0.0218 & 0.0212 & 0.0212 & 0.020001 \\
\hline & 2 & 0.0200 & 0.0002 & 0.0218 & 0.0212 & 0.0212 & 0.020001 \\
\hline & 3 & 60.0700 & 0.6007 & 62.2074 & 64.3424 & 64.3415 & 64.40350 \\
\hline & 4 & 18.8800 & 0.1888 & 17.0280 & 15.8600 & 15.8602 & 15.98760 \\
\hline & 5 & 13.8800 & 0.1388 & 12.4784 & 11.7590 & 11.7591 & 11.82440 \\
\hline & 6 & 4.9500 & 0.0495 & 5.4335 & 5.1064 & 5.1064 & 5.117290 \\
\hline & 7 & 2.0100 & 0.0201 & 2.3239 & 2.1842 & 2.1842 & 2.185620 \\
\hline & 8 & 0.3700 & 0.0037 & 0.4851 & 0.7058 & 0.7063 & 0.441642 \\
\hline Corrente 2 & Topo & 4.8300 & 0.0483 & 5.0500 & 5.2200 & 5.2200 & 5.224100 \\
\hline \multirow[t]{8}{*}{ Composição \% } & $1^{*}$ & - & - & 0.0365 & 0.0342 & 0.0341 & 0.032223 \\
\hline & $2^{*}$ & - & - & 0.0365 & 0.0342 & 0.0341 & 0.032223 \\
\hline & 3 & 99.9400 & 0.9994 & 99.9076 & 99.9005 & 99.8982 & 99.91560 \\
\hline & 4 & 0.0200 & 0.0002 & 0.0019 & 0.0260 & 0.0260 & 0.020002 \\
\hline & 5 & 0.0000 & 0.0001 & $1.40 \times 10^{-6}$ & 0.0076 & 0.0076 & $7.37 \times 10^{-7}$ \\
\hline & $6 \#$ & 0.0000 & - & 0.0000 & 0.0000 & 0.0000 & 0.0000 \\
\hline & $7 \#$ & 0.0000 & - & 0.0000 & -0.0003 & 0.0000 & 0.0000 \\
\hline & $8 \#$ & 0.0000 & - & 0.0000 & -0.0021 & 0.0000 & 0.0000 \\
\hline Corrente 3 & Fundo & 3.2200 & 0.0322 & 3.3900 & 3.1900 & 3.1900 & 3.19260 \\
\hline \multirow[t]{8}{*}{ Composição \% } & $1 \#$ & 0.0000 & - & 0.0000 & 0.0000 & 0.0000 & 0.0000 \\
\hline & $2 \#$ & 0.0000 & - & 0.0000 & 0.0000 & 0.0000 & 0.0000 \\
\hline & 3 & 6.3900 & 0.0639 & 6.0590 & 6.2790 & 6.2790 & 6.29467 \\
\hline & 4 & 40.1800 & 0.4018 & 42.3599 & 41.7155 & 41.7167 & 42.1155 \\
\hline & 5 & 29.3100 & 0.2931 & 31.0627 & 30.9481 & 30.9488 & 31.1728 \\
\hline & 6 & 14.4200 & 0.1442 & 13.5258 & 13.4447 & 13.4449 & 13.4908 \\
\hline & 7 & 6.5500 & 0.0655 & 5.7849 & 5.7511 & 5.7509 & 5.76197 \\
\hline & 8 & 3.1400 & 0.0314 & 1.2076 & 1.8617 & 1.8597 & 1.16431 \\
\hline
\end{tabular}

*variáveis não medidas; \#variáveis fixas (valores conhecidos e isentos de reconciliação).

$\mathrm{Na}$ Tabela 1 observa-se os resultados. Entretanto, para avaliar as estratégias de solução apresentadas utilizaram-se os critérios de Fobj e SARES, apresentados na Tabela 2. 
Tabela 2 - Avaliação das estratégias de RD para o estudo de caso 1.

\begin{tabular}{ccccc}
\hline Critérios & Crowe & Simpson & PNL & EMSO \\
\hline Fobj & $1.40 \times 10^{+5}$ & $1.26 \times 10^{+5}$ & $1.25 \times 10^{+5}$ & $\mathbf{1 . 0 6 \times 1 0 ^ { + 5 }}$ \\
SARES & $1.75 \times 10^{-1}$ & $7.84 \times 10^{-1}$ & $7.74 \times 10^{-1}$ & $\mathbf{1 . 2 9}^{-1} \mathbf{1 0}$ \\
\hline
\end{tabular}

$\mathrm{Na}$ Tabela 2 verifica-se que o simulador EMSO obteve os melhores resultados.

\subsection{Resultado do Estudo de Caso 2}

Na Tabela 3 são apresentados os resultados obtidos neste trabalho e por Wang et al. (2004), bem como os valores exatos, medidos e respectivos desvios-padrão.

Tabela 3 - Resultados da RD para o estudo de caso 3.

\begin{tabular}{|c|c|c|c|c|c|}
\hline & \multirow[b]{2}{*}{ Exato } & \multirow[b]{2}{*}{ Medido } & \multirow[b]{2}{*}{$\sigma_{\mathrm{i}}$} & \multicolumn{2}{|c|}{ Valores Reconciliados } \\
\hline & & & & Wang et al. (2004) & EMSO \\
\hline $\mathrm{x} 1$ & 308 & 300.4 & 7.7 & 308.7 & 308.33 \\
\hline $\mathrm{x} 2$ & 77 & 74 & 1.925 & 75.9 & 75.8324 \\
\hline $\mathrm{x} 3$ & 77 & 75.7 & 1.925 & 77.6 & 77.5324 \\
\hline $\mathrm{x} 4$ & 77 & 75.3 & 1.925 & 77.2 & 77.1324 \\
\hline $\mathrm{x} 5$ & 77 & 76 & 1.925 & 78 & 77.8324 \\
\hline $\mathrm{x} 6$ & 62 & 62.4 & 1.55 & 62.2 & 62.1168 \\
\hline $\mathrm{x} 7$ & 82 & 84.1 & 2.05 & 83.7 & 83.6043 \\
\hline $\mathrm{x} 8$ & 82 & 81.4 & 2.05 & 81 & 80.9043 \\
\hline $\mathrm{x} 9$ & 82 & 82.2 & 2.05 & 81.8 & 81.7043 \\
\hline $\mathrm{x} 10$ & 308 & 309.4 & 7.7 & 308.7 & 308.33 \\
\hline $\mathrm{x} 11$ & 8.5 & 8.3 & 0.2125 & 8.3 & 8.27828 \\
\hline $\mathrm{x} 12$ & 5.5 & 5.7 & 0.1375 & 5.7 & 5.6909 \\
\hline $\mathrm{x} 13$ & 30 & 29.7 & 0.75 & 29.4 & 29.4286 \\
\hline $\mathrm{x} 14$ & 20 & 20.7 & 0.5 & 20.5 & 20.5795 \\
\hline $\mathrm{x} 15$ & 14 & 14.3 & 0.35 & 14.2 & 14.241 \\
\hline x16 & 230 & 314.8 & 5.75 & 230.6 & 230.112 \\
\hline $\mathrm{x} 17$ & 57.5 & 58.3 & 1.4375 & 57.5 & 57.4029 \\
\hline $\mathrm{x} 18$ & 57.5 & 58.3 & 1.4375 & 57.5 & 57.4029 \\
\hline $\mathrm{x} 19$ & 57.5 & 59.9 & 1.4375 & 59.1 & 59.0029 \\
\hline $\mathrm{x} 20$ & 57.5 & 57.2 & 1.4375 & 56.5 & 56.3029 \\
\hline x21 & 230 & 180.5 & 5.75 & 230.6 & 230.112 \\
\hline $\mathrm{x} 22$ & 12 & 11.9 & 0.3 & 11.9 & 11.8965 \\
\hline $\mathrm{x} 23$ & 49 & 48.5 & 1.225 & 48.6 & 48.4412 \\
\hline $\mathrm{x} 24$ & 38 & 36.6 & 0.95 & 36.7 & 36.5646 \\
\hline $\mathrm{x} 25$ & 21 & 21.4 & 0.525 & 21.4 & 21.3892 \\
\hline $\mathrm{x} 26$ & 110 & 140.6 & 2.75 & 112 & 111.82 \\
\hline SSE & - & - & - & 18.59 & 16.84 \\
\hline
\end{tabular}


Na Tabela 3 observa-se os resultados obtidos no procedimento de RD com dados com múltiplos erros grosseiros nas variáveis x16, x21 e x26, para a estratégia MT-NT (Wang et al., 2004) e o estimador robusto Normal Contaminada do pacote de RD do simulador EMSO. Fica claro que os maiores ajustes ocorrem nas variáveis com erros grosseiros e que o simulador EMSO obteve o melhor resultado global, uma vez que apresentou o menor valor para SSE.

Em ambos os estudos de caso utilizou-se o otimizador do IPOPT presente no EMSO.

\section{CONCLUSÃO}

Este trabalho apresentou um estudo sobre reconciliação de dados em estado estacionário em colunas de destilação com base em dois exemplos apresentados na literatura. No primeiro o simulador EMSO foi comparado às estratégias para solução de sistemas bilineares: Matriz de Projeção, Técnica de Simpson e Programação Não Linear, em uma coluna debutanizadora. No segundo o simulador EMSO foi comparado à estratégia de detecção de múltiplos erros grosseiros MT-NT, utilizando-se o estimador robusto Normal Contaminada, em um conjunto de 7 colunas em série. Em ambos os estudos o pacote computacional EMSO obteve melhores resultados, comprovando a eficiência e robustez deste simulador.

\section{REFERÊNCIAS}

CROWE, C. M. Reconciliation of Process Flow Rates by Matrix Projection, Part II: The Nonlinear case. AIChE J., v. 32, p. 616-623, 1986.

FARZI, A.; MEHRABANI-ZEINABAD, A.; BOOZARJOMEHRY, R. B. Data reconciliation: Development of an object-oriented software tool. Korean J. Chem. Eng., v. 25, p. 955-965, 2008.

KISTER, H. Z. Distillation-Design. EUA: Editora McGraw-Hill, 1992.

NARASHIMHAN, S.; JORDACHE, C. Data Reconciliation and Gross Error Detection: An Intelligent Use of Process Data. Texas: Editora Gulf Professional Publishing, 2000.

ÖZYURT, D.B.; PIKE, R.W. Theory and practice of simultaneous data Reconciliation and gross error detection for chemical process. Comput. Chem. Engng., v.28, p. 381-402, 2004.

PRATA, D. M.; SCHWAAB, M.; LIMA, E. L.; PINTO, J. C. Simultaneous Robust Data Reconciliation and Gross Error Detection through Particle Swarm Optimization for an Industrial Polypropylene Reactor. Chem. Eng. Sci., v. 65, p. 4943-4954, 2010.

RAO, R. R.; NARASIMHAN, S. Comparison of Techniques for Data Reconciliation of Multicomponent Processes. Ind. Eng. Chem. Res., v. 35, p. 1362-1368, 1996.

SIMPSON, D. E.; EVERETT, M. G.; VOLLER, V. R. An efficient algorithm for mineral processing data adjustment. Int. J. Miner. Process, v. 31, p. 73-96, 1991.

SOARES, R. P.; SECCHI, A. R. EMSO: Envirolnment for Modelling, Simulation and Optimisation. Comput. Aided Chem. Eng., v. 14, p. 947-952, 2003.

WANG, F.; JIA, X.; ZHENG, D.; YUE, J. An improved MT-NT method for gross error detection and data reconciliation. Comput. Chem. Eng., v. 28, pp. 2189-2192, 2004. 